\title{
Nonlinear behavior of acoustic waves in combustion chambers-II
}

\author{
F. E. C. CUI.ICK \\ California Instilute of Technology, Pasiden: ( A $911(0), U$.S A.
}

(Received 15 September 1975; revised 4 March 1976)

\begin{abstract}
The approximate analysis developed in Part 1 of this work is applied to several specific problems. Ote purpose is to illustrate the use of the formalism, and a second is to demonstrate the validity of the method by comparing results with numerical solutions, obtained elsewhere, for the "exact" equations. A simple problem is treated first, the decay of a standing wave in a box containing a mixture of gas and suspended particles; one example of the steepening of an initially sinusoidal wave in pure gas is included. Viscous losses on an inert surface are treated essentially according to classical linear theory; recent experimental resulis are used as the basis for incorporating approximately the influence of nonlinear heat transfer in unsteady flow. All of the preceding results are combined in calculations of two examples of unstable motions in a solid propellant rocket molor and in a $T$-burner.
\end{abstract}

\section{Introduction}

IN THE first part of this work (Culick, 1976), hereafter referred to as I, a general formalism is described for treating both linear and nonlinear processes assciciated with acoustic waves in combustion chambers. The method is suitable for studying many problems involving waves in chambers; a few relatively simple examples are described here. Ultimately, specific results can be obtained only by numerical integration of a set of coupled nonlinear first-order ordinary differential equations. One of the purposes of this paper is to show how the representations of certain kinds of physical processes are incorporated prior to numerical computations. For convenience in reading, a brief summary of the development of the analysis is given below. Some of the formulas required in subsequent discussion are included, but Part I will frequently be referred to in later sections.

It is simplest to treat problems involving no flow or combustion. The analysis then describes the development of waves in a closed chamber. Some examples for the attenuation of waves in a gas containing particles are given in Section 2; a special case is the steepening of sinusoidal waves into triangular waves in a pure gas containing no particles. Some comparisons are made with "exact" numerical solutions to the conservation equations for one-dimensional motions. For the conditions examined, the approximate analysis appears to be quite accurate, and is, of course, considerably cheaper to use.

The viscous losses at inert surfaces are often important; thesc are treated in Section 3. For linear behavior, the classical results are derived in a slightly different manner from that usually given. Some recent global measurements of losses are incorporated to provide a means of estimating the effect of nonlinear behavior. 
Preliminary to tecating unstahle waves in eombustion ehambers, atl approximate way of acconnting for tratusient combustion is discussed in Section 4. Finally, in Section 5 unstable motions in a small solid propellant rocket motor and in a $T$-burner are analyzed. The discussion is intended only to provide illustrations of the method, and 1101 as an exhaustive account. Once again, comparisons with numerical solutions are made.

All of the examples discussed here involve only longitudinal modes, for which the natural frequencies are integral multiples of the fundamental. Formulas valid for any geometry, i.e. any set of normal modes, have been deduced hy culick (1975), but to date no numerical results have been obtained. Eventually, an important application of the approximate analysis will be for three-dimensional problems which are prohibitively expensive to solve by more exact numerical techniques. It is a necessary preliminary step to demonstrate the validity of the approximate analysis, as far as possible, by the sorts of comparisons with "exact" solutions discussed here.

In broad outline, the formalism of the approximate analysis is developed in the following three steps. The conservation equations are first manipulated (Section? of 1) to produce a nonlinear inhomogeneous wave equation for the pressure disturbances, with an inhomogeneous boundary condition:

$$
\begin{aligned}
\frac{1}{\bar{a}^{2}} \frac{\partial^{2} p^{\prime}}{\partial t^{2}}-\nabla^{2} p^{\prime} & =-h \\
\hat{n} \cdot \nabla p^{\prime} & =-f .
\end{aligned}
$$

The functions $h$ and $f$ contain representations of all perturbations of the classical acoustics problem for the actual geometry being considered. Two important points should be noted. First, the speed of sound $\bar{a}$, and other physical properties not explicitly shown, are those for a two-phase (or in general a multiphase) mixture. This is a consequence of combining the original conservation equations to give a description of the flow as that for a single average fluid. Second, the forms of the functions $h$ and $f$ depend on what order of perturbations are considered. The procedure is based on expansion in two small parameters, the Mach numbers $\vec{M}$ and $M^{\prime}$ of the average and fluctuating flows. Terms in $h$ and $f$ which are linear in $M^{\prime}$ also depend linearly on $\bar{M}$; these produce results for problems of stability. In the present work, nonlinear terms from the gasdynamics are carried to order $M^{\prime 2}$ only and are independent of $\bar{M}$. Other nonlinear terms will arise, for example, in the treatment of nonlinear particle damping discussed in Section 2.

The second step is based on expansion of the unsteady pressure and velocity fields in normal modes $\psi_{i}(r)$ of the chamber, with time-varying amplitudes $\eta_{i}(t)$ :

$$
\begin{aligned}
& \frac{p^{\prime}}{p_{o}}=\sum_{i=0}^{\infty} \eta_{i}(t) \psi_{i}(\mathbf{r}) \\
& \mathbf{u}^{\prime}=\sum_{i=1}^{\infty} \frac{\dot{\eta}_{i}(t)}{\bar{\gamma} k_{i}^{2}} \nabla \psi_{i}(\mathrm{r})
\end{aligned}
$$


where $k_{i}=a\left(s_{i}\right.$ is the wavenumber for the ith mode. After (1.1) has heen multiplied by $\psi_{n}(r)$ and integrated over the volume of the chamber, the ordinary differential equation for $\eta_{n}(t)$ can be deduced:

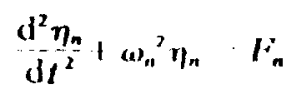

with

$$
F_{n}=\frac{a^{2}}{p_{1} E_{n}^{2}}\left\{\int t h \mathrm{~d} V+\oiint \psi_{n} f \mathrm{~d} S\right\}
$$

Finally, in the third step. the method of averaging is applied to reduce the second order equations (1.5) to first order equations. The amplitudes are written

$$
\eta_{n}=A_{n}(t) \sin \omega_{n} t+B_{n}(t) \cos \omega_{n} t
$$

and averaging over the time interval $(t, t+\tau)$ leads to the first order equations for the $A_{n}(t)$ and the $B_{n}(t)$ :

$$
\begin{aligned}
& \frac{\mathrm{d} A_{n}}{\mathrm{~d} t}=\frac{1}{\omega_{n} \tau} \int_{t}^{++\tau} F_{n} \cos \omega_{n} t^{\prime} \mathrm{d} t^{\prime}, \\
& \frac{\mathrm{d} B_{n}}{\mathrm{~d} t}=\frac{-1}{\omega_{n} \tau} \int_{2}^{\mathrm{t}+} F_{n} \sin \omega_{n} t^{\prime} \mathrm{d} t^{\prime} .
\end{aligned}
$$

For the special case of longitudinal modes $\left(\omega_{n}=n \omega_{1}\right)$, the interval of averaging is taken as the period of the fundamental mode, $\tau=\tau_{2}=2 \pi / \omega_{1}$, and the limits on the integrals may be changed to $\left(0, \tau_{1}\right)$ for any function $F_{n}$ arising in problems of interest in this work.

If only the nonlinearities arising from the gasdynamics are shown, the force $F_{n}$ has the general form

$$
F_{n}=-\sum_{i=1}^{\infty}\left[D_{n} \dot{\eta}_{i}+E_{n i} \eta_{i}\right]-\sum_{i=1}^{\infty} \sum_{i=1}^{\infty}\left[A_{n i} \dot{\eta}_{i} \dot{\eta}_{i}+B_{n i} \eta_{i} \eta_{j}\right]
$$

For longitudinal modes, $F_{n}$ simplifies considerably (see Part 1 ) and the equations (1.8) and (1.9) are

$$
\frac{\mathrm{d} A_{n}}{\mathrm{~d} t}=\alpha_{n} A_{n}+\theta_{n} B_{n}+\frac{\beta n}{2} \sum_{i=1}^{\infty}\left[A_{i}\left(A_{n-i}-A_{i-n}-A_{n+i}\right)-B_{i}\left(B_{n-i}+B_{i-n}+B_{n-i}\right)\right]
$$

$$
\frac{\mathrm{d} B_{n}}{\mathrm{~d} t}=\alpha_{n} B_{n}-\theta_{n} A_{n}+\frac{\beta n}{2} \sum_{i=1}^{\infty}\left[A_{i}\left(B_{n-i}+B_{i, n}-B_{n+i}\right)+B_{i}\left(A_{n-i}-A_{i-n}+A_{n-i}\right)\right] .
$$


The linear coeflicients $\alpha_{n}, \theta_{n}$ are related lo the coeflicients $\left./\right)_{. . . .} / V_{n .}$ in $(1.10)$ hy

$$
x_{n} \cdot{ }_{2}^{1} l_{n+\cdots} \cdot \theta_{n} \cdot \frac{1}{2\left(u_{n}\right.} l_{n, n}
$$

This is a useful rule, because $\alpha_{n}$ is the familiar growth constant for linear motions, and $\theta_{n}$ is proportional to the shift of frequency.

\section{An approximation to the linear and nonlinear attenuation}

of waves by gas/particle interactions

Particularly in solid propellant rockets using metallized propellants, but in other systems as well, some of the combustion products appear in the form of liquid or solid particles. The viscous interactions between the particles and the gas may, under suitable conditions, provide a significant dissipation of energy. It is of ten the case that the Reynolds number hased on the particle diameter is outside the range in which Stokes' law is valid; it is necessary to use a more realistic representation of the drag force. This introduces another nonlinear influence in the general problem.

Let $F_{n}{ }^{(p)}$ denote that part of $F_{n}$ in eqn (1.6), representing the influences of inert particles. The terms involved are those containing $\delta F_{p}^{\prime}$ and $\delta Q_{p}^{\prime}$, the fluctuations of (2.8) and (2.9) in I. By tracing the development in I from (2.10) and (2.11) to (3.16), with $H$ defined by $(3.15)$ in I, one finds that the terms in cuestion are

$$
F_{n}^{(p)}=\frac{\bar{\gamma}}{\rho_{c} E_{n}^{2}} \int \frac{1}{\epsilon}\left[\frac{1}{\bar{a}^{2}} \frac{\bar{R}}{\bar{C}_{n}} \frac{\partial}{\partial t}\left(\delta Q_{p}^{\prime}+\delta \mathbf{u}_{p}^{\prime} \cdot \mathbf{F}_{p}\right) \psi_{n}+\delta F_{p}^{\prime} \cdot \nabla \psi_{n}\right] \mathrm{d} V
$$

Formulas for $\delta F_{p}^{\prime}$ and $\delta Q_{p}^{\prime}$ can be found only by solving the equations of motion for the particles. Numerical calculations (I.evine and Culick, 1972; 1974) have shown that for many practical cases, nonlinear interactions are likely to be important. The approximate analysis here will be based on the nonlinear laws for the force $F_{p}$ and heat transfer $Q_{p}$ used in those works:

$$
\begin{aligned}
& F_{p}=\rho_{1} \frac{18 \mu}{\rho_{s} \sigma^{2}}\left(u_{r}, \quad u\right)\left[1+\frac{1}{6} R e^{2 / 3}\right] \\
& \left.Q_{1} \cdots \rho_{n} \frac{12 C_{n} \mu}{\operatorname{Prp}_{s}\left(T_{n}\right.}, \quad T\right)\left[1+0.23 \operatorname{Pr}^{0 \cdots} \mathrm{Ke}^{0 " s]}\right.
\end{aligned}
$$

where

$$
\operatorname{Re}=\frac{\rho_{\mu .} . v_{\mu}}{\mu}\left|\mathbf{u}_{p}-\mathbf{u}\right|
$$

Hereafter, the real flow will be treated only in a local approximation so that the particle motions may be treated as one-dimensional; interactions between particles are assumed to be negligible. Results will be given for steady-state harmonic motion: short term transient motions are neglected. Details of the analysis maty be found in the report by Culick (1975). The linear part of the force. 
for longitudinal modes, is

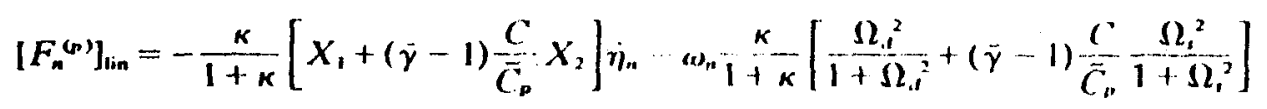

where

$$
\begin{aligned}
& X_{1}=\left(\omega_{n} \Omega_{d}\right) /\left(1+\Omega_{d}\right) \\
& \left.X_{2}\right)\left(\omega_{n} \Omega_{1}\right) /\left(1+\Omega \Omega_{a}^{3}\right) .
\end{aligned}
$$

A point not to be forgotten when this result is used is that the entire approximate analysis is founded on an iteration/perturbation procedure. This strictly requires that the force $F_{n}$ on the right hand side of the oscillator equation $\ddot{\eta}_{n}+\omega_{n}{ }^{2} \eta_{n}=F_{n}$ be small, in the sense of the inequality

$$
F_{n} \ll \omega_{n}^{2} \eta_{n}
$$

Application of this constraint to the first term of (2.25) gives the requirement

$$
\frac{\kappa}{1+\kappa} \frac{\Omega_{d}}{1+\Omega_{d}^{2}} \ll 1
$$

Because the procedure followed here is different from that used by Temkin and Dobbins (1966) to treat this problem, so also are the limits of validity. Their simplified results, which exhibit the same frequency dependence as $(2.20)$ and (2.21), are valid for $\kappa \ll 1$. Consequently, the present formulation should have the advantage of being applicable over a broader range of particle loading.

By applying the rule (1.13) above, one finds for the linear contributions from gas/particle interactions:

$$
\begin{gathered}
\alpha_{n}^{(n)}=-\frac{1}{2}\left(\frac{\kappa}{1+\kappa}\right)\left[X_{1}+(\bar{\gamma}-1) \frac{C}{\bar{C}_{p}} X_{2}\right] \\
\theta_{a}^{(p)}=\frac{\omega_{n}}{2}\left(\frac{\kappa}{1+\kappa}\right)\left[\frac{\Omega_{d}{ }^{2}}{1+\Omega_{d}{ }^{2}}+(\bar{y}-1) \frac{C}{\bar{C}_{p}} \frac{\Omega_{t}{ }^{2}}{1+\Omega_{i}{ }^{2}}\right] .
\end{gathered}
$$

Recent numerical results reported by Levine and Culick (1974) have shown that the result (2.9) is quite good for smaller particles, and if the frequency is not too high. Beyond limits which are presently not well-defined, the Reynolds number (2.4) becomes too large for the linear drag and heat transfer laws to be accurate. Further comments on the accuracy and some examples are given below.

The problem of analyzing nonlinear particle motions is avoided here. A correct treatment would involve solving the equations of motion with the nonlinear laws (2.2) and (2.3). A very much simpler course is taken here: the linear solutions are used to compute the next approximation in an iteration procedure (Culick, 1975). 
Fventually the terms to be incorporated in the ecpuations for the $A_{n}$ and $B_{n}$ are found to be:

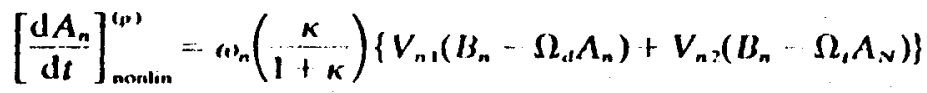

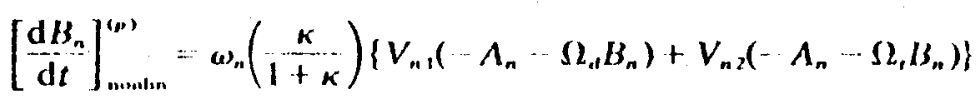

where

$$
\begin{gathered}
V_{n 1} \ldots \frac{1.396}{\pi} W_{n 1}\left(A_{n}{ }^{2}+B_{n}{ }^{2}\right)^{n .3 n !}, \\
V_{n 2} \frac{0.918}{\pi} W_{n 2}\left(A_{n}{ }^{2}+B_{n}{ }^{2}\right)^{n .273} \\
W_{n 1}=\frac{2}{\pi}(0.698)\left(1+\Omega_{d}\right)^{\epsilon_{1}{ }^{2}} K_{1}\left(\frac{\bar{a} X_{1}}{\bar{\gamma} \omega_{n}}\right)^{\epsilon_{1}}\left(\frac{X_{1}}{\left(\omega_{n} \Omega_{d}\right.}\right) \\
W_{n 2}=\frac{2}{\pi}(0.644)\left(1+\Omega_{d}{ }^{2}\right)^{\epsilon_{2}{ }^{2}}(\bar{\gamma}-1) \frac{C}{\bar{C}_{p}} K_{2}\left(\frac{\bar{a} X_{1}}{\bar{\gamma} \omega_{n}}\right)^{\epsilon_{2}}\left(\frac{X_{2}}{\omega_{n} \Omega_{t}}\right) .
\end{gathered}
$$

A few results have been obtained for the attenuation of a standing wave initially excited in a box of length $L$ containing a gas/particle mixture. These have been carried out for direct comparison with the numerical results reported by Levine and Culick (1974). First, one case will be given in some detail. The particle diameter is $2.5 \mu$, the particle loading is $\kappa=0.36$ and the frequency based on the equilibrium speed of sound $\bar{a}$ is $800 \mathrm{~Hz}$ in each se. The material and thermodynamic properties are listed below:

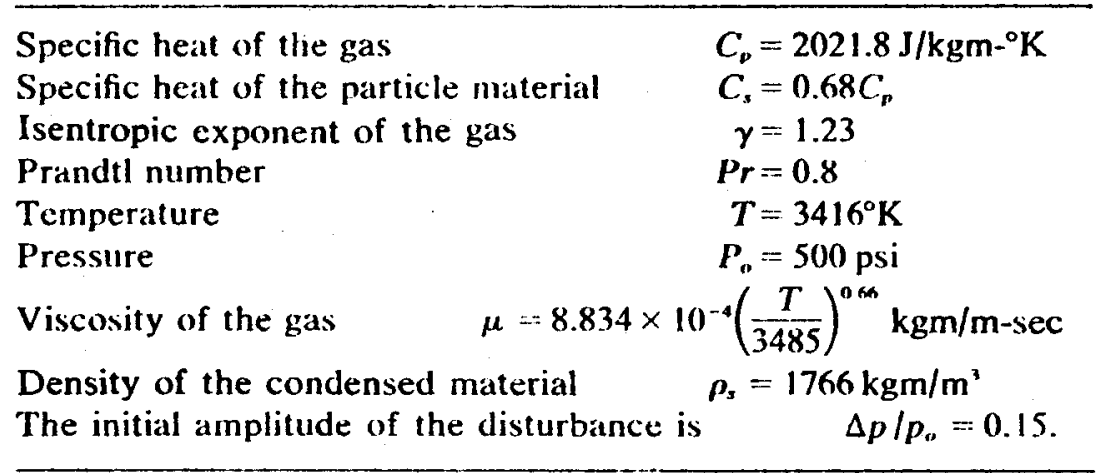

Figures 2.1 and 2.2 show the waveforms obtained from the approximate and numerical analyses. The approximate results are the solutions to eqns (1.11) and (1.12) with the linear coefficients $\alpha_{n}, \theta_{n}$ given by (2.9) and (2.10) and with additional nonlinear terms by (2.11) and (2.12). The period used to normalize the time scale is the period $2 L, / \bar{a}$ for linear waves based on the speed of sound of the 


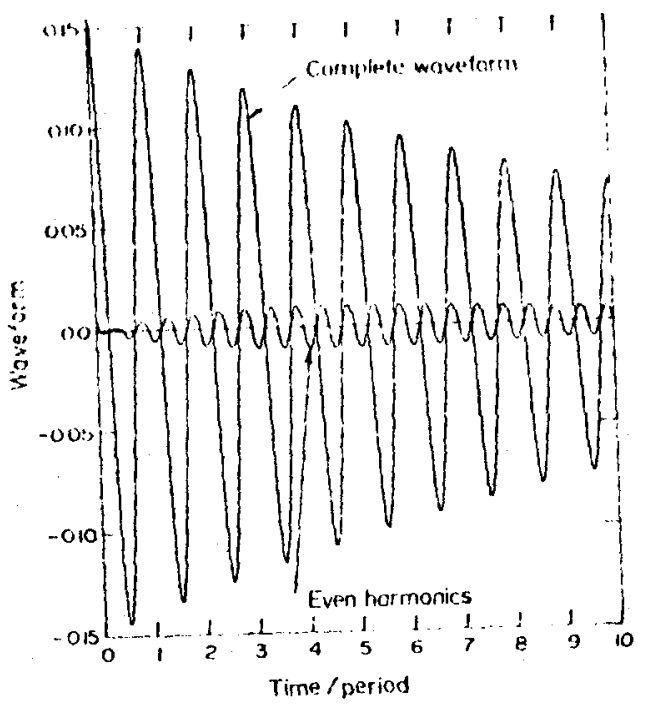

Fig. 2.1. Altenuation by $2.5-\mu$ particles at $800 \mathrm{~Hz}$ according to the approximate analysis, $\Delta p(0) / p_{\circ}:=0.15$.

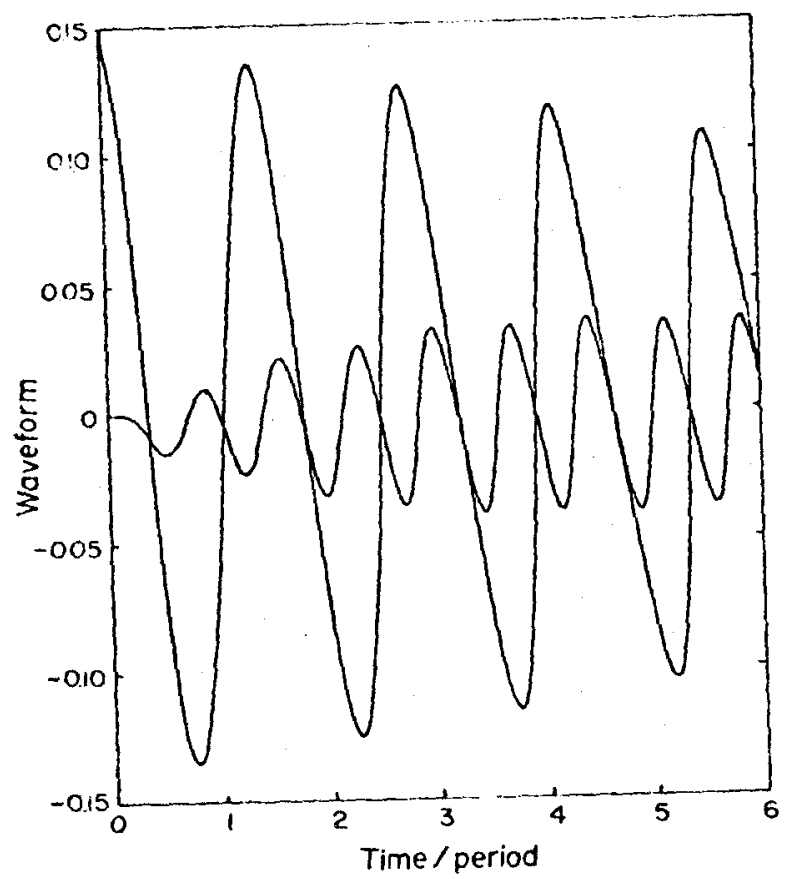

Fig. 2.2. Attenuation by $2.5-\mu$ particles at $800 \mathrm{~Hz}$ according to the numerical analysis, $\Delta p(0) / p_{0}=0.15$. 
mixture. The similarities between the wateforms and the generation and decay of the even harmonics is apparent, but there is a difference in the relative phases between the even harmonics and the totil witveform. "This seems to arise almost entirely in the first cycle of the oxcillation; it maty be due to detaits of the numerical routines and the way in which the computations hegin. This difference may therefore not reflect at genuine difference between the approximate and "exact" antilyses.

A more quantitative measure of the hehavior is the instintaneous value of the decay constant, $\alpha_{w}$. calculitted for successive peaks of the waveform. This history of $\alpha_{p}$ is shown in Fig. 2.3. Further remarks on the behavior of $\alpha_{p}$ may be found in

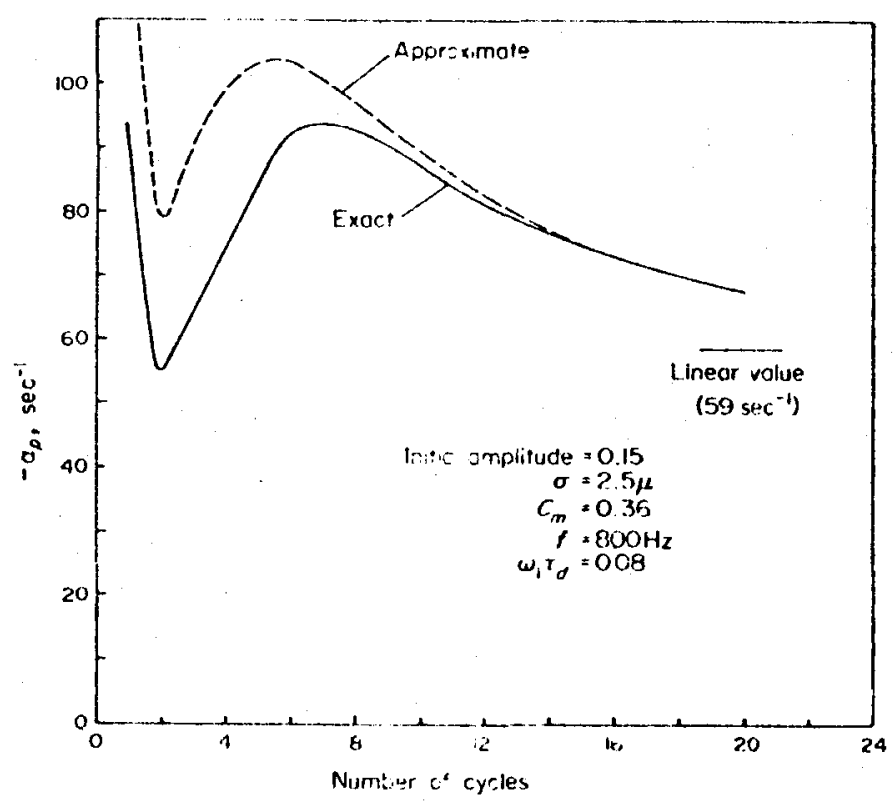

Fig. 2.3. The decay constant for the cases shown in Figs. 2.1 and 2.2.

Section 7 of the report by Levine and Culick (1974). The purpose here is only to demonstrate that the approximate analysis does give fairly reasonable results for this case. However, there are limitations. not yet clearly defined, which arise from the approximate treatment of the nonlinear acoustics as well as the gas/particle interactions.

The linear behavior used here, described essentially by velocity and temperature lags, is in fact quite restrictive. Although it is not apparent from the analyses presented here, the work reported by Levine and Culick shows that the results are accurate only when $\omega \tau_{d}$ is small. If this condition is met, then $\delta u_{p}$ and $\delta T_{p}$ are relatively small, which is consistent with the general nature of the perturhation analysis used here. For the $2.5-\mu$ particles, $\omega \tau_{d} \approx 0.08$ at $800 \mathrm{~Hz}$.

A case exhibiting more nonlinear behavior is shown in Fig. 2.4. The waveform calculated with the approximate analysis is shown for 30- $\mu$ particles at $1500 \mathrm{~Hz}$ 


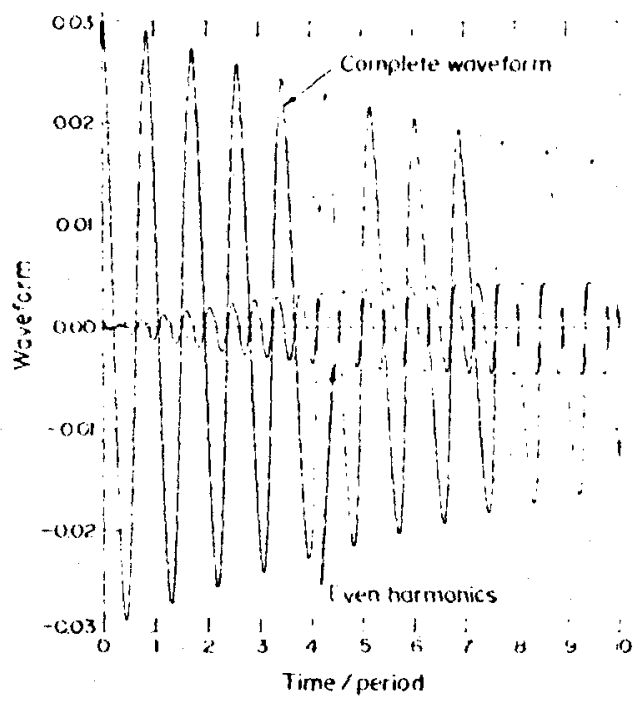

Fig. 2.4. Attenuation by $30-\mu$ particles at 1500$) H z$ according to the approximate analysis; $\Delta p(0) / p_{0}=0.03$.

$\left(\omega \tau_{d} \approx 25.8\right)$. The value of $\alpha_{p}$ is $-70 \mathrm{sec}^{-1}$ at an amplitude of $1 \%$, and the numerical analysis gives $-148 \mathrm{sec}^{-1}$. Note that the fractional error between the approximate and numerical results for $\alpha_{p}$ increases with increasing $\omega \tau_{d}$. The greater amount of harmonic content present when larger particles are considered is due mostly to the reduced drag and hence reduced attenuation at the higher frequencies. The results from the approximate analysis can be improved for higher values of $\omega \tau_{d}$ by using different functions $X_{1}, X_{2}$ instead of (2.6) and (2.7).

Wave propagation in a gas/particle mixture is dispersive; the speed of propagation depends on the frequency and particle size, through the parameter $\omega \tau_{d}$, as well as on the amount of condensed material present. For the problems treated here, the length of the box, and hence the wavelength of the waves, is fixed. Consequently, a change in the speed of sound is reflected as a shift of frequency. The periods of the waveforms shown in Figs. 2.1, 2.2 and 2.4 are not exactly equal to the period based on the equilibrium speed of sound. It is obvious from the figures that the frequency shift increases with $\omega \tau_{d}$. In Section 5 of Part I, it is shown that to first order, the frequency shift due to linear dispersion is $\delta f=-\theta / 2 \pi$; here, $\theta$ is given by $(2.10)$ for the $n$th mode. The actual frequency change is dominated by $\theta_{1}$; values of $\theta_{1}$ and $\delta$ calculated from the waveforms for several cases are given in Table 2.1 , a summary of the computations discussed here.

These results serve to demonstrate that the nonlinear generation of harmonics has substantial influence of the detailed character of the attenuation of waves. The rather close agreement between the approximate results and the numerical calculations under conditions when the approximation to the gas/particle interaction is more accurate suggests that the approximate treatment of the fluid mechanics is realistic, at least for moderate amplitudes. 


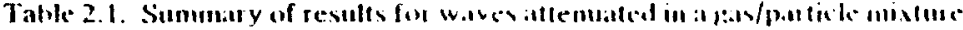

\begin{tabular}{|c|c|c|c|c|c|c|c|c|}
\hline $\begin{array}{c}\text { Particle } \\
\text { Diameter } \\
(\mu)\end{array}$ & $\begin{array}{c}f . \\
(H z)\end{array}$ & $\begin{array}{c}f \\
(H z .)\end{array}$ & $f$ & w & $\begin{array}{c}s f \\
(H / L)\end{array}$ & $\begin{array}{l}n_{1} \\
2 r\end{array}$ & $\begin{array}{l}\left(\left\langle x_{p}\right)_{1 . *}\right. \\
(s c c \text { ' })\end{array}$ & $\begin{array}{l}\left(m_{n}\right)_{1}= \\
\left(\mathrm{sec}^{\prime}\right)\end{array}$ \\
\hline \multicolumn{9}{|l|}{2.5} \\
\hline Approximale & $x(x)$ & 954 & $8(n)$ & 0.08 & 1 & 0.7 .4 & 59 & 61 \\
\hline Numerical & 800 & 954 & 8111 & 0.08 & 1 & $\ldots$. & -58 & 59 \\
\hline Approximale & $15(0)$ & 1788 & 1505 & 0.15 & 5 & 4.8 & 203 & -204 \\
\hline Numerical & 1500 & 1788 & 1504 & 0.15 & 4 & $\cdots$ & -201 & -204 \\
\hline \multicolumn{9}{|l|}{10} \\
\hline Approximate & $15(10$ & 1788 & 1695 & 2.4 & 195 & 189.9 & 509 & -530 \\
\hline Numerical & 1500 & 1788 & 1740 & 2.8 & 240 & - & .509 & -870 \\
\hline \multicolumn{9}{|l|}{30} \\
\hline Approximate & 1500 & 1788 & 1740 & 216 & 240 & 224.1 & 67 & $\cdots 70$ \\
\hline Numerical & $15(x)$ & 1788 & 1787 & 25.8 & 287 & - & $\therefore 69$ & -118 \\
\hline
\end{tabular}

f. frequency hatsed an the equilibrium

f. frequency based on the speed for the gis only, $a_{a} \cdots(1 \gamma-1) c_{p} T l^{1 / 2}$

$f$ frequency of the calculated wate

$\delta f=:-f$.

$\left(\alpha_{p}\right)_{\text {hin }}$ decay constant for linear waves

$\left(\alpha_{p}\right)_{x}$ decay constant for the calculated wave at approximately $1 \%$ amplitude

Note. Small differences arise in some quantities $\left(\omega \pi_{d}\right.$ and $\left.\left(\alpha_{p}\right)_{\text {hin }}\right)$ which should have the same values when calculated by the approximate and numerical methods. This is due to a small difference in the value of some property, most probably the viscosity.

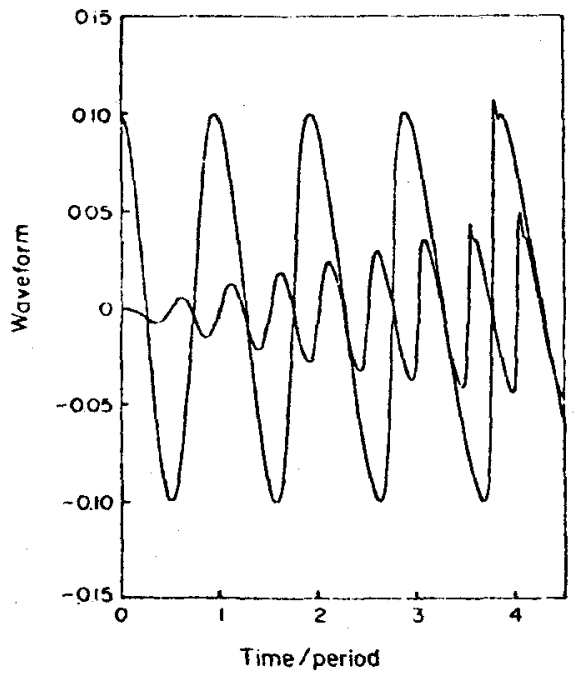

Fig. 2.5. Steepening of a standing wave in a pure gas according to the numerical analysis; $f=900 \mathrm{~Hz}$.

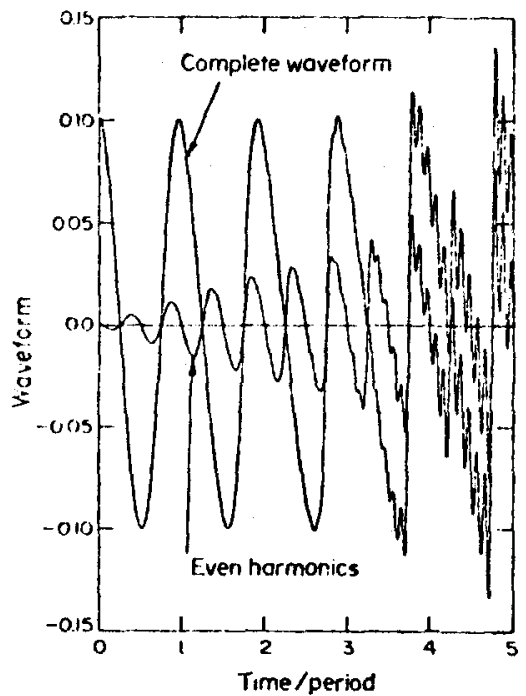

Fig. 2.6. Steepening of a standing wave in a pure gas according to the approximate analysis with ten modes accounted for; $f=900 \mathrm{~Hz}$. 
From the point of view of reducing data for the attenuation by particle/gas interactions, it is annoying that the value of the decay constant changes so much as the waves dic out (cf. leig. 2.3). It is possible that if the higher harmonics ate filtered from the waveform, the hehavior of or for the first harmonic alone maly not be so extreme. Calculations have not been done (o) check this point.

Perhaps the simplest check of the fluid mechanics alone is calculation of the hehavior of a wave in a hox with no particles present. In this cane, the wave must of course steepen, eventually forming a shock wave. Neither the exact nor approximate analyses can accommodate strong shock waves, hut the initial period of development may usefully he examined. Figure 2.5 shows the exact result, and Fig. 2.6 shows the result of the approximate analysis when ten modes are accounted for. Again, the qualitative agreement is quite good. As one would anticipate, the period of the wave decreases as the amplitude increases. For both the approximate and numerical analyses, obvious distortion of the peak occurs at about the fourth cycle. (The sharp jags in Fig. 2.5 may be due in part of the numerical routine.)

\section{An approximation to nonlinear viscous losses on an inert surface}

There are two reasons for examining the influence of viscous stresses and heat transfer at an inert surface: these processes can be significant siabilizing infuences; and in the presence of oscillations, the average heat transfer increases substantially. The second is a nonlinear effect which has on occasion caused serious structural problems, particularly in liquid rocket motors. The main purpose of this section is to show one way of incorporating some experimental results within the analysis developed earlier. As part of the argument, the more familiar linear results will also be recovered.

The viscous stresses and heat transfer at a surface are, of course, associated with a boundary layer, but they can be accommodated here by suitable interpretation of the force $F$ and heat source $Q$ in the equations developed in Part I. It is only those terms which are required in this discussion, so the wave equation for the pressure fluctuation is simply

$$
\frac{\partial^{2} p^{\prime}}{\partial t^{2}}-\bar{a}^{2} \nabla^{2} p^{\prime}=\frac{\bar{R}}{\bar{C}_{v}} \frac{\partial Q^{\prime}}{\partial t}-\bar{a}^{2} \nabla \cdot \mathbf{F}^{\prime}
$$

The boundary condition is

$$
\hat{n} \cdot \nabla p^{\prime}=\hat{n} \cdot \mathbf{F}^{\prime}
$$

For this problem, then, the equation for the amplitude of the $n$th harmonic is

$$
\ddot{\eta}_{a}+\omega_{n}^{2} \eta_{n}=\frac{\vec{\gamma}}{\rho_{o} \bar{a}^{2} E_{n}^{2}}\left\{\frac{\vec{R}}{\bar{C}_{0}} \frac{\mathrm{d}}{\mathrm{d} t} \int Q^{\prime} \psi_{n} \mathrm{~d} V-\bar{a}^{2} \int \mathbf{F}^{\prime} \cdot \nabla \psi_{n} \mathrm{~d} V\right\}
$$

The heat source $Q^{\prime}$ is taken here to be associated with the heat flux vector $\bar{q}^{\prime}$, and 
the force $\mathbf{F}$ with the viscous stress tensor $\ddot{\tau}$ :

$$
Q^{\prime}=\nabla \cdot \mathbf{q}^{\prime} \quad \mathbf{F}=\nabla \cdot \overline{\boldsymbol{r}}^{\prime}
$$

Both $q^{\prime}$ and $\bar{\tau}^{\prime}$ are significantly non-zero only in thin regions near the boundary. Then if $y$ denotes the coordinate normal to the wall, measured positive inward, $d=\mathrm{d} y \mathrm{~d} S$ and

$$
Q^{\prime}=\frac{\partial q_{y}^{\prime}}{\partial y} \quad F^{\prime}=\frac{\partial \tau_{y}^{\prime}}{\partial y}
$$

The conventions used here are that $q_{y}^{\prime}$, the component of $q^{\prime}$ normal to the surface, is positive for heat transfer to the wall, and $\mathbf{F}^{\prime}$, being parallel to the surface, is positive when the force tends to accelerate the gas. In the volume integrals of (3.3), $\psi_{n}$ and $\nabla \psi_{n}$ are essentially independent of $y$, so one can write

$$
\begin{gathered}
\int Q^{\prime} \psi_{n} \mathrm{~d} v \approx \iint \mathrm{d} S \psi_{n} \int_{0}^{x} \frac{\partial q_{y}^{\prime}}{\mathrm{d} y} \mathrm{~d} y=-\iint q_{w}^{\prime} \psi_{n} \mathrm{~d} S \\
\int \mathbf{F}^{\prime} \cdot \nabla \psi_{n} \mathrm{~d} v \approx \iint \mathrm{d} S \nabla \psi_{n} \cdot \int_{0}^{\infty} \frac{\partial \tau_{y}^{\prime}}{\partial y} \mathrm{~d} y=-\iint \tau_{w}^{\prime} \cdot \nabla \psi_{n} \mathrm{~d} S .
\end{gathered}
$$

Here, the surface stress is $\tau_{w}^{\prime}=-\mu\left(\partial \mathbf{u}^{\prime} \partial^{\prime} \partial y\right)_{w}$, where $\mathbf{u}^{\prime}$ is the velocity fluctuation parallel to the surface, so

$$
-\iint \tau_{w}^{\prime} \cdot \nabla \psi_{n} \mathrm{~d} S=\iint\left(\mu \frac{\partial \mathbf{u}^{\prime}}{\partial y}\right)_{w} \cdot \nabla \psi_{n} \mathrm{~d} S
$$

Only the linear stress will be treated, but both linear and nonlinear contributions to the heat transfer will be accommodated by writing

$$
q_{w}^{\prime}=\left(k \frac{\partial T^{\prime}}{d y}\right)_{n}+\vec{h}\left(T_{n}^{\prime}-T_{w}^{\prime}\right)
$$

Owing to the thermal inertia of the watl, $T_{w}^{\prime}=0$; the temperature fluctuation $T_{\infty}^{\prime}$ far from the wall is that associated with the acoustic field. The average heat transfer coefficient, $\bar{h}$, will be assumed in a manner described below, to depend on the amplitude of the acoustic field.

Equation (3.3) can now be written

$$
\begin{gathered}
\ddot{\eta}_{n}+\omega_{n}^{2} \eta_{n}=-\frac{\bar{\gamma}}{\rho_{a} E_{n}^{2}} \iint\left(\mu \frac{\partial u^{\prime}}{\partial y}\right)_{w} \cdot \nabla \psi_{n} \mathrm{~d} S-\frac{\bar{R} / \bar{C}_{\mathrm{v}} \mathrm{d}}{p_{o} E_{n}^{2}} \frac{\mathrm{d} t}{\mathrm{~d} t} \int\left(k \frac{\partial T^{\prime}}{\mathrm{d} y}\right)_{w} \psi_{n} \mathrm{~d} S \\
-\frac{\bar{R} / \bar{C}_{\mathrm{v}} \mathrm{d}}{p_{o} E_{n}^{2}} \iint \bar{h} T_{x_{1}}^{\prime} \psi_{n} \mathrm{~d} S .
\end{gathered}
$$

First, calculation of the linear parts will be outlined. The known solutions for 
the velocity and temperalure fluctuations in a sinusoidal acoustic field are

$$
\begin{aligned}
& u^{\prime}=\hat{n}\left[1-c^{A y}\right] e^{m+\cdots t}
\end{aligned}
$$

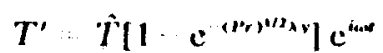

where $\hat{u}, \hat{T}$ are the anplitudes far from the wall, and

$$
\lambda=\frac{1}{\delta}(1+i) \delta \quad \sqrt{ }(2 i / \omega) .
$$

Thus, for purely harmonic ascillations,

$$
\begin{aligned}
& \left(\mu \frac{\partial u^{\prime}}{\partial y}\right)_{w}=\frac{\mu}{\delta}(1+i) \hat{u} \mathrm{e}^{i \omega \omega t} \\
& \left(k \frac{\partial T^{\prime}}{\partial y}\right)_{w}=\frac{k}{\delta}(P r)^{1 / 2}(1+i) \hat{T} \mathrm{e}^{i \omega t} .
\end{aligned}
$$

These formulas are for steady oscillations. For transient motions, exact results can be obtained, but those are too complicated for the purposes here. Instead, the following approximation is used. Note that for sinusoidal motions, $i$ may be replaced by $\omega^{-1} \partial / \partial t$. This replacement will be made, and $\hat{\imath}$ and $\hat{T}$ will be taken to stand for the values associated with each mode. For the $n$th mode one eventually finds that (3.10) may be written

$$
\ddot{\eta}_{n}+\omega_{n}{ }^{2} \eta_{n}=-2 \alpha_{n}^{\left({ }^{(1)}\right.}\left(\dot{\eta}_{n}-\omega_{n} \eta_{n}\right)-\frac{\vec{R} / \overline{C^{\prime}}}{p_{o} E_{n}{ }^{2}}\left(\frac{\bar{y}-1}{\bar{\gamma}}\right) T_{0} \frac{\mathrm{d}}{\mathrm{d} t} \iint \bar{h} \eta_{n} \psi_{n}^{2} \mathrm{~d} S
$$

where

$$
\alpha_{n}^{(\omega)}=\frac{\left(\omega_{n} \bar{\nu} / 2\right)^{1 / 2}}{2(1+\kappa)} \frac{1}{E_{n}^{2}} \iint\left[\left(\frac{\nabla \psi_{n}}{k_{n}}\right)^{2}+\frac{\bar{\gamma}-1}{V(P r)} \iint \psi_{n}^{2} \mathrm{~d} S .\right.
$$

It has been assumed that the motion far from the wall is isentropic, so $T_{\infty}^{\prime}=(\bar{\gamma}-1)\left(T_{\omega} p^{\prime} / \bar{\gamma} p_{0}\right)$. For a longitudinal mode in a straight cylindrical tube,

$$
\iint\left(\frac{\nabla \gamma_{n}}{k n}\right)^{2} \mathrm{~d} S=\iint \psi_{n}^{2} \mathrm{~d} S=\pi D L / 2
$$

and with $x=0,(3.14)$ becomes the familiar result for the decay constant for a standing longitudinal wave:

$$
\alpha_{n}^{(u)}=\frac{2}{D} \sqrt{ }\left(\frac{\omega_{n} \nu}{2}\right)\left(1+\frac{\bar{\gamma}-1}{\sqrt{ }(P r)}\right)
$$

The treatment of the remaining term in (3.13) rests on appeal to some recent experimental results. Perry and Culick (1974) have reported measurements of the 
time-and space-averaged values of the heal transfer coeflicient in at T-burner with propellant dises at the ends. Denote this coeflicient by $\langle\vec{h}\rangle$. The data could be quite well represented by the expression

$$
\frac{\langle\bar{h}\rangle \delta}{k}=0.044 R e^{11}
$$

where

$$
\text { Re.. } \frac{|\hat{p}| \operatorname{lon} \delta}{\mu a}
$$

The symbol $|\hat{p}|_{m}$ represents the maximum amplitude of the pressure fluctuation, namely that measured at the end of the $T$-hurner; this is equal to $p_{0}\left|\eta_{n}\right|$ for the $n$th mode.

In the absence of any other information, two assumptions will be made. First, it is reasonable to assume that the local time-averaged heat transfer coefficient is also proportional to the square root of the pressure amplitude, as (3.15) shows for the space-averaged value. Hence, for the $n$th mode,

$$
\bar{h}=K_{n},\left.\psi_{n} \eta_{n}\right|^{1 / 2}
$$

where $K_{n}$ is a constant to be determined. The sccond assumption is that (3.17) is valid for all modes. From the definitions of $\bar{h}$ and $\langle\bar{h}\rangle$,

$$
\langle\bar{h}\rangle=\frac{1}{S} \iint \bar{h} \mathrm{~d} S=\frac{1}{L} \int_{0}^{c} \bar{h} \mathrm{~d} z
$$

for a cylinder. It follows from (3.15)-(3.18) that the constant $K_{n}$ is given as

$$
K_{n}=0.044 \frac{k V\left(p_{o}\right)}{V(\mu \bar{a})}\left(\frac{\omega_{n}}{2 \bar{\nu}}\right)^{1 / 4}\left[\frac{1}{L} \int_{0}^{I .}\left|\psi_{n}\right|^{1 / 2} \mathrm{~d} z\right]^{-1}
$$

The integral has the value

$$
\frac{1}{L} \int_{0}^{L}\left|\psi_{n}\right|^{1 / 2} \mathrm{~d} z=\frac{1}{L} \int_{0}^{1}\left|\cos k_{n} z\right|^{1 / 2} \mathrm{~d} z=0.765
$$

so

$$
K_{n}=0.0575 \frac{k V\left(p_{0}\right)}{V(\mu \bar{a})}\left(\frac{\omega_{n}}{2 \bar{\nu}}\right)^{1 / 4}
$$

Note that the mode shape cos $\left(k_{n} z\right)$ is used to obtain (3.19) because the data was taken in a uniform tube. The assumptions introduced above imply that the result is supposed to be valid for a local surface element whatever may be the mode in the chamber.

After the method of averaging has been applied, and various integrals have 
heen evaluated (see ('ulick, 1975), cquations for $A_{n}$ and $B_{, .}$, with ouly the viscous losses shown are

$$
\begin{aligned}
& \frac{\mathrm{d} A_{n}}{\mathrm{~d} t} \cdot \alpha_{n}{ }^{(1)}\left(A_{n}-B_{n}\right)-0.457 H_{n} A_{n}\left(A_{n}{ }^{2}+B_{n}{ }^{2}\right)^{1 / 4} \\
& \frac{\mathrm{d} B_{n}}{\mathrm{~d} t}-\cdots x_{n}{ }^{(n)}\left(A_{n}+B_{n}\right)-0.4571 H_{n} B_{n}\left(A_{n}{ }^{2}+B_{n}{ }^{2}\right)^{1 / 4}
\end{aligned}
$$

In the special circumstance when these equations are applied to watves in a cylindrical chamber with an inert lateral boundary,

$$
J_{n}=\frac{1.836}{R_{c}}
$$

where $R_{c}$ is the radius of the chamber; and

$$
0.457 H_{n}=\frac{1.143}{R_{r}} \frac{\bar{\gamma}-1}{\operatorname{Pr}_{\bar{\gamma}}^{1 / 2}}\left[\left(\frac{\bar{\nu}}{10^{-4}}\right)\left(\frac{f_{n}}{1000}\right)\left(\frac{\bar{a}}{1000}\right)^{2}\right]^{1 / 4}
$$

where the units are: $R_{c}, \mathrm{~m} ; \vec{\nu}, \mathrm{m}^{2} / \mathrm{sec} ; f_{n}, \mathrm{~Hz} ;$ and $\vec{a}, \mathrm{~m} / \mathrm{sec}$.

\section{An approximation to the influence of transient surface combustion}

The source of the energy for unsteady motions in a combustion chamber is the combustion itself. Interactions between combustion and unsteady fluid motions are commonly represented by some sort of frequency response function, which is particularly appropriate for studying the stability of sinusoidal disturbances. The use of a response function is not limited to linear problems, but the problem is much more complicated for nonlinear behavior. Only the simplest representation of the influence of unsteady combustion processes will be covered here. Elementary results for linear response to harmonic pressure variations form the basis. Certain of the features of truly transient behavior will be ignored in the interest of obtaining formulas which are clear and inexpensive to use. Only surface combustion will be discussed, but the same strategy may be used for combustion within the volume.

The influence of surface combustion is contained in $\mathscr{R}$, defined by eqn (3.18) of Part I. It follows from (3.16) and (4.1) of I that the corresponding contribution to the force $F_{n}$ in $(1.6)$ is

$$
F_{n}^{(c)}=\frac{\bar{\gamma}}{\rho_{\Delta} E_{n}^{2}} \frac{\partial}{\partial t} \oiint \mathscr{A} \psi_{n} \mathrm{~d} S
$$

To simplify the discussion, consider only one of the pieces of $\mathscr{R} ;$ e.g. let $\delta_{\perp}=1$, $\delta_{\|}=0$, so $(3.18)$ of $I$ is

$$
\mathscr{R}=\rho_{0} u_{b}^{\prime}+\bar{u}_{b} \frac{p^{\prime}}{a_{0}{ }^{2}}=(1+\kappa)\left(m_{b}^{\prime}+\bar{m}_{h} \frac{\Delta T^{\prime}}{T_{0}}\right) .
$$


It is best here also lo atvoid the complications associalled with a condensed phase: set $\kappa=0$, so the following results apply to propellatils not colltaining metal.

There exists a class of analyses, discussed by ('ulick (1968). which produces a formula for $\dot{m}_{b} / \dot{m}_{b}$, the fluctuation of mass flux due to a simusoidal variation of pressure. This is a linear result, $\dot{m}_{n} / m_{n}$ being proportiomal to the pressure fluctuation:

$$
\frac{\hat{m}_{b}}{\bar{m}_{b}}=K_{b} \frac{\hat{p}}{p_{o}}=\left|R_{b}^{(r)}+i R_{b}^{(i)}\right| \frac{\hat{p}}{p_{o}} .
$$

The response function, $R_{b}$, is a complex function of frequency,

$$
R_{b}=-\frac{n A B}{\lambda+\frac{A}{\lambda}-(1+A)+A B},
$$

in which $A$ is proportional to the activation energy for the surface reaction, and $B$ depends on both $A$ and the heat released by the surface reaction. Out of the same analyses, one can extract the formula for $\Delta \hat{T} / T_{o}$; and with these results, (4.2) becomes, for sinusoidal motions,

$$
\begin{aligned}
\hat{\mathscr{R}} & =\rho_{o} \bar{u}_{b}\left[\left(1+\frac{\bar{T}_{i}}{T_{o}} \frac{C}{C_{p}} \frac{A B}{E}\right) K_{b}-\left(n \frac{\bar{\Gamma}_{s}}{T_{o}} \frac{C}{C_{p}} \frac{A B}{E}+\frac{\gamma-1}{\gamma}\right)\right] \frac{\hat{p}}{p_{o}} \\
& =\rho_{o} \bar{u}_{b}\left[\mathscr{R}^{(r)}+i \mathscr{R}^{(i)}\right] \frac{\hat{p}}{p_{o}} .
\end{aligned}
$$

Note that $\mathscr{R}^{(r)}$ and $\mathscr{R}^{(i)}$ are dimensionless functions of the frequency and the other parameters; if nonisentropic temperature fluctuations are ignored, $\mathscr{R}_{i}^{(r)}=R_{b}^{(r)}\left(\omega_{i}\right)$ and $\mathscr{R}_{i}^{(i)}=R_{b}^{(i)}\left(\omega_{i}\right)$.

Now the formula (4.5) is, by construction, for steady sinusoidal variations only. As in the preceding section, the replacement $i \rightarrow \omega^{\prime \prime} \partial / \partial t$ will be made in (4.5), and the result is assumed to apply to all modes. Thus, $\hat{p} / p_{0}$, stands for $\eta_{i} \psi_{i}$, and for an arbitrary pressure field expanded in the form (1.3), of in (4.1) will hereafter be taken as

$$
\mathscr{R}=\rho_{0} \ddot{u}_{n} \sum_{i=1}\left[\left(\mathscr{R}_{i}^{(n)}+\frac{1}{\omega_{i}} \mathscr{R}_{i}^{(n)} \frac{\partial}{\partial t}\right] \eta_{i} \psi_{i-}\right.
$$

The subscript ()$_{i}$ on $\mathscr{R}{ }_{i}^{(r)}$ and $\mathscr{R}_{i}^{(i)}$ means that each function is evaluated at the frequency of the ith mode; these quantities can be calculated from (4.5) and (4.4).

The force (4.1) can now easily be evaluated, and the rule (1.13) gives directly the contributions from surface combustion to $\alpha_{n}^{(c)}$ and $\theta_{n}^{(c)}$ to be used in (1.11) and (1.12):

$$
\begin{aligned}
& \alpha_{n}{ }^{(c)}=\frac{\gamma \bar{u}_{b}}{2 E_{n}{ }^{2}} \mathscr{R}_{i}{ }^{(r)} \oiint \psi_{n}{ }^{2} \mathrm{~d} S \\
& \theta_{n}^{(c)}=-\frac{\mathscr{R}_{n}^{(i)}}{\mathscr{R}_{n}^{(r)}} \alpha_{n}^{(c)} \text {. }
\end{aligned}
$$


The same procedure can be applied to propellants containing metal: only some details are changed to account for $\kappa \neq 0$.

A similar approximation can be used for handling combustion within the volume of a chamber as one finds in liquid rockets, thrust augmentors, and solid rockets exhibiting residual combustion. Although other contributions will in general arise, associated with mass and momentum exchange, the direct contribution of energy release is represented by the terms containing $Q$ and $w_{w}$, in eqn (2.2) of $I$. The dynamical behavior of combustion within the volume may, for example, be represented by some sort of response function; the well-known $n-\tau$ model developed by Crocco ct al. is a special form. In any case, the contributions to the individual harmonics can be approximated as surface combustion was handled above. No results for bulk combustion have been obtained.

\section{Application to the stability of longitudinal}

modes in a rocket motor

It has already heen shown that the nonlinear terms simplify consideratily for the case of longitudinal modes. This siluation will be treated here for one example. Some preliminary results were reported by I cvine and Culick (1974). Application to large motors was examined by Culick and Kumar (1974). The discussion here is to demonstrate how the approximate analysis can be used to study practical configurations and to provide a limited comparison with the more exact numerical results reported elsewhere.

For all the calculations reported in this section, the following material and thermodynamic properties are used:

Specific heat of the gas

Specific heat of the particle material Thermal diffusivity of the propellant Prandtl number of the gas

Viscosity of the gas

Isentropic exponent of the gas Linear burning rate of the propellant Density of the propellant Density of the condensed material

$$
\begin{gathered}
C_{\mathrm{p}}=2021.8 \frac{\mathrm{J}}{\mathrm{kgm}-{ }^{\circ} \mathrm{K}} \\
C_{s}=0.68 C_{\rho} \\
\kappa=10^{-7} \mathrm{~m}^{7} / \mathrm{sec}^{2} \\
P r=0.8 \\
\mu=8.834 \times 10^{-4}(T / 3485)^{0.05} \frac{\mathrm{kgm}}{\mathrm{m}-\mathrm{sec}} \\
\gamma=1.23 \\
\bar{r}=0.00812\left(p_{u} / 500\right)^{11} \mathrm{~m} / \mathrm{sec} \\
\rho_{c}=4000 \mathrm{kgm} / \mathrm{m}^{3} \\
\rho_{s}=1766 \mathrm{kgm} / \mathrm{m}^{3}
\end{gathered}
$$

Because the nonlinear acoustics terms represented by the series in (1.11) and (1.12) are given explicitly, the first step in the analysis consists in evaluating the contributions to the linear coefficients $\alpha_{n}$ and $\theta_{n}$. Four contributions to linear stability are included; arising from the nozzle, the condensed material in the gas phase, the surface combustion processes, and the one-dimensional approximation to inelastic acceleration of flow issuing from the lateral surface ("flow turning"). The formulas for the corresponding values of $\alpha_{n}$ are 
Nozzle

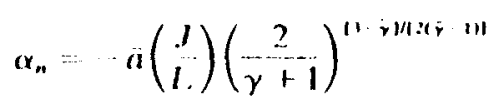

Paricles

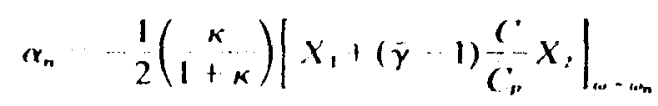

Flow Turning

$$
\alpha_{n}=-\frac{1}{2} \bar{u}_{n}\left(\frac{S_{b}}{V}\right)
$$

\section{Combustion}

$$
\alpha_{n}=\frac{1}{2} \bar{\gamma} \bar{u}_{b}\left(\frac{S_{b}}{V}\right) R_{b}{ }^{(n)}
$$

The formula (5.1) is based on quasi-steady behavior; the velocity fluctuations are in-phase with pressure fluctuations at the nozzle entrance. Hence, the response function has no imaginary part and, as shown by eqn (4.10), the value of $\theta_{n}$ for the nozzle is zero. The term representing flow-turning is the last one of (3.17) in I; there is no corresponding $F_{\text {si }}$, so $\theta_{n}$ is also zero for flow turning. The only non-zero values of $\theta_{n}$ are for the gas/particle interactions and combustion; the first is given by (2.12) and the second by use of (4.8) and (5.4):

Particles

$$
\theta_{n}=\frac{\omega_{n}}{2}\left(\frac{\kappa}{1+\kappa}\right)\left[\frac{\Omega_{d}^{2}}{1+\Omega_{d}{ }^{2}}+(\bar{\gamma}-1) \frac{C}{\bar{C}_{p}} \frac{\Omega_{1}^{2}}{1+\Omega_{t}{ }^{2}}\right]_{\omega-\omega_{n}}
$$

\section{Combustion}

$$
\theta_{n}=-\frac{1}{2} \bar{\gamma} \bar{u}_{h}\left(\frac{S_{b}}{V}\right) R_{b}^{(i)}
$$

The two examples treated here were discussed in Section 7.4 of Culick and Levine (1974), where the result of the numerical analysis is given. Each is for a motor having a cylindrical hore and length 23.5 in. The mean pressure, port area, and throat area differ and are given below:

\begin{tabular}{lcc}
\hline & (a) & (b) \\
\hline Length (in.) & 23.5 & 23.5 \\
Port area (in $\left.{ }^{2}\right)$ & 3.33 & 4.73 \\
Throat area (in $\left.{ }^{2}\right)$ & 0.439 & 0.562 \\
Pressure (psia) & 1568 & 1412 \\
\hline
\end{tabular}


These are the first and last cases given in Table 7.3 by Culick and Levine (1974). The fundamental frequency is $900 \mathrm{H} z$.

For both cases, the particle diameter is assumed to be $2.0 \mu$ and the mass fraction is $\kappa=0.36$. The combustion response is taken to be the representation (7.4) given above, with $A=6.0$ and $B=0.56$. Because the pressures are different, so are the flame temperatures in the two cases; for (a), $T_{1}=3525^{\circ} \mathrm{K}$ and for (b), $T_{f}=3515^{\circ} \mathrm{K}$. The small difference has only minor influence on the results.

With the above values, and the formulas (5.1)-(5.4), waves for case (a) are found to be stable. The values of the decay constants for the first five modes are given in Table 5.1. The numerical calculations produced an unstable wave which, with an initial amplitude of $5 \%$ (fundamental mode) eventually reached a limiting amplitude of $4.2 \%$. In view of the successful comparison for the cases treated in Section 2 for attenuation by particle damping, and because the representation of the combustion response is the same in the approximate and numerical analysis, a likely source of the difference in the results is the behavior of the nozzle.

Table 5.1. Values of $\alpha_{n}$ and $\theta_{n}$ for three cases of unsteady motions in a small motor

\begin{tabular}{rrrr}
\hline & $\begin{array}{c}\text { Case (a) } \\
\left(\sec ^{~}\right)\end{array}$ & $\begin{array}{r}\text { Case (ad) } \\
\left(\sec ^{1}\right)\end{array}$ & \multicolumn{1}{c}{$\begin{array}{c}\text { Case (b) } \\
\left(\mathrm{sec}^{-1}\right)\end{array}$} \\
\hline$\alpha_{2}$ & -18.5 & 8.0 & -9.1 \\
$\alpha_{2}$ & -369.3 & -342.8 & -334.8 \\
$\alpha_{3}$ & -610.1 & -583.6 & -566.5 \\
$\alpha_{4}$ & -915.9 & -889.4 & -871.4 \\
$\alpha_{3}$ & -1289.2 & -1262.7 & -1244.0 \\
$\theta_{1}$ & 12.9 & 12.9 & 64.0 \\
$\theta_{2}$ & 46.8 & 46.8 & 34.9 \\
$\theta_{3}$ & -29.3 & -29.3 & -35.6 \\
$\theta_{3}$ & -131.0 & -131.0 & -135.0 \\
$\theta_{3}$ & -280.0 & -280.0 & -283.0 \\
\hline
\end{tabular}

In the numerical analysis, the calculations for the entire two-phase flow were carried out to the nozzle throat. The influence of the nozzle is buried in the results and its contribution to attenuation cannot be determined. For the approximate analysis, the influence of the nozzle is represented as a surface admittance function evaluated at the nozzle entrance. The result (5.1) is strictly valid for quasi-steady behavior of a gas only; it has been extended to the case of two-phase flow by using the value of $\bar{\gamma}$ for the mixture. The point is that there is reason to expect that the contribution of the nozzle is different in the two analyses.

As a means of comparing the analyses, the value of the attenuation constant associated with the nozzle is chosen to obtain the same limiting amplitude as found in the numerical analysis. This procedure rests on the observation that the values of the limiting amplitudes are quite sensitive to the values of the linear coeflicients $\alpha_{n}, \theta_{n}$.

Not a very large change is required. Equation (5.1) gives $\alpha_{n}=-153.25 \mathrm{sec}^{-1}$. If this is changed to $-126.75 \mathrm{sec}^{-1}$, then $\alpha_{1}=8.00$ and the limiting amplitude is 
about $4.2 \%$. The values of the $\alpha_{n}$ and $\theta_{n}$ for five modes are given in Table 5.1, for the case denoted (aa). Figure 5.1 shows the amplitudes of the five harmonics considered; the functions $A_{n}$ and $B_{n}$ are shown in Figs. 5.2 and 5.3. The waveform at limiting amplitude shows no obvious evidence of distortion from the fundamental mode.

The attenuation by the nozzle is assumed to vary linearly with the ratio $J$ of throat area to port area. having the value $-126.75 \mathrm{sec}$ ' when $J$ has the value (0.132) for case (a). Then with the other required data given above, the

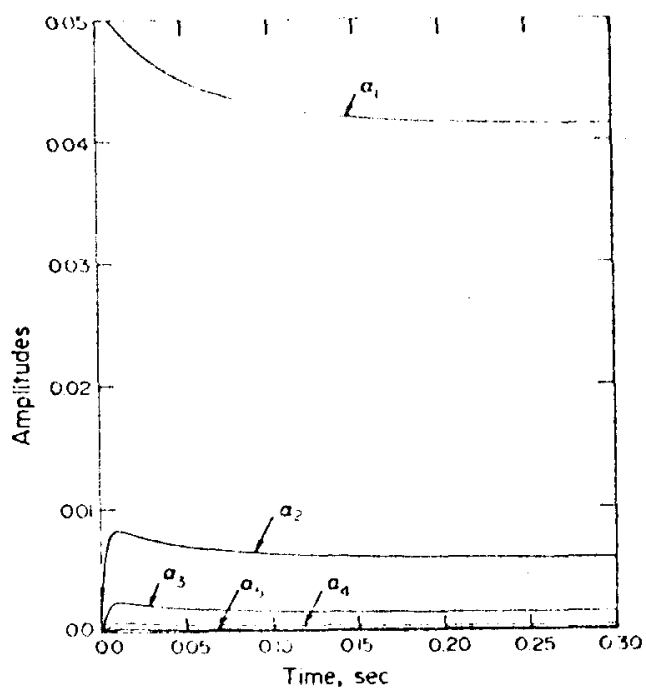

Fig. 5.1. Amplitudes for unstuble oscillations in a motor, case (a) of Table 5.1.

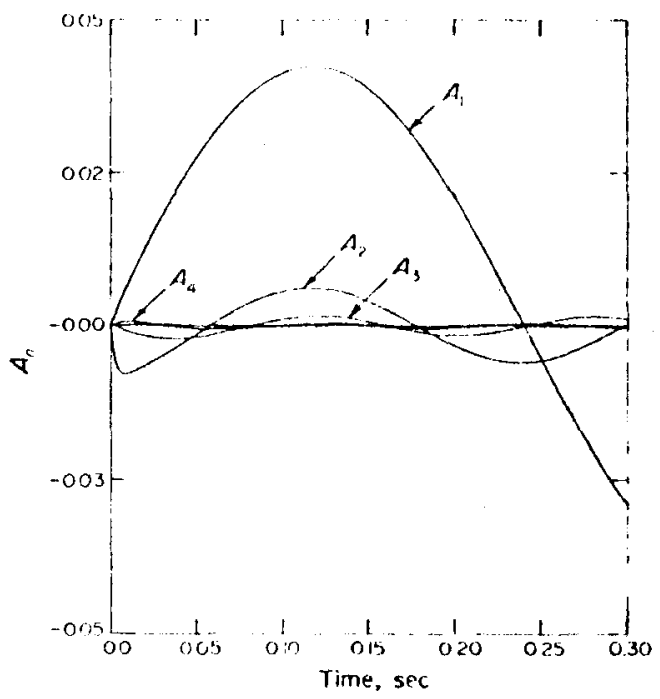

Fig. 5.2. The functions $A_{a}(t)$ for case (ad) of Table S.1. 


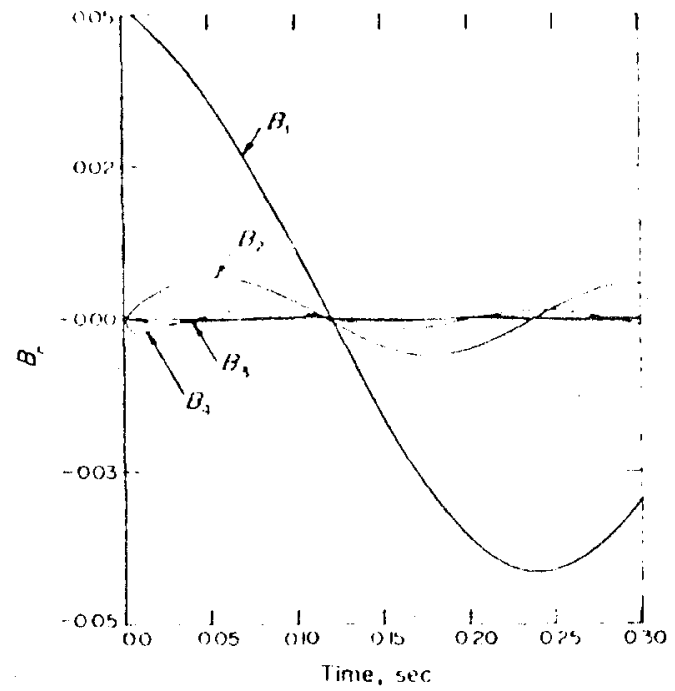

Fig. 5.3. The functions $B_{n}(t)$ for casc (aa) of Table 5.1 .

approximate analysis applied to case (b) produces the results shown in the last column of Table 5.1. The initial disturbance is stable, the decay constant for the first harmonic being $\alpha_{1}=-9.12 \mathrm{sec}^{-1}$. After twenty cycles $(0.02222 \mathrm{sec})$ the amplitude is roughly $3.5 \%$ according to the approximate analysis. The numerical analysis gave an amplitude of $3.02 \%$ after twenty cycles. It appeared that the wave may have stabilized at a limiting amplitude, but the calculation was not carried further. In view of the slow decay found with the approximate analysis, it may be that the conclusion based on the numerical analysis, for only 20 cycles, is incorrect, based on incomplete results. If a true limit was indeed reached, then of course the approximate analysis gives the wrong result. A limit of $3 \%$ would be reached only if $\alpha_{1}$ is positive, having a value less than 8.00 .

That nonlinear influences are in fact active, even at such relatively small amplitudes, is easily established. Consider a wave having a decay constant equal to $-9.12 \mathrm{sec}^{-1}$ and a frequency of $900 \mathrm{~Hz}$. According to linear behavior, the amplitude would be $4.08 \%$ after 20 cycles if the initial amplitude is $5 \%$. The more rapid decay shown by the nonlinear analysis is evidently due to the transfer of energy, through the nonlinear processes, from the fundamental oscillation to higher harmonics which are then attenuated much more rapidly. Nonlinear particle damping was included in the approximate analysis but, as one should expect for the small amplitudes arising in these examples, its influence is negligibly small.

An example of longitudinal modes in a $T$-burner has been treated elsewhere by Culick (1976).

Acknowledgments--This work was supported partly by Hercules, Inc., Magna, Utih; by the Aerojet Solid Propulsion Co.. Sacramento, California; and by the National Aeronautics and Space Administration under the Jet Propulsion I,aboratory Contract NAS 7-100. 


\section{References}

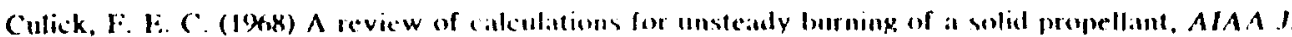
6(12), $2241 \cdot 2255$.

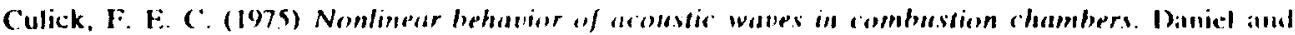
Florence Cinguenheim Jet Propulsion center, California Instilute of Teclonology. Pasadenit. Californiat.

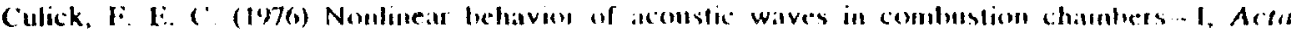
Astronastica $1,9 / 10,714,14$

Culick. F. F. C. and Kumar, K. N. (1974) (ambustion instability in large solid rocket motors, loth JANNAF Combustion Mesting, Newpont, Rhode Island.

Culick, F. E. C. and I evine, J. N. (197.1) Comparison of approximate and numerical analyses of nonlinear combustion instability, AIA 12 th Aerospace Sciences Meeting. Washington, D.C. AIAA Paper No. 74-201.

Levine, J. N. and Culick, F. H. (. (1972) Numerical analysis of nonlinear longitudinal combusfion instability in metallized propellant solid rocket motors. Ultrasystems, Inc., Irvine, Calif., Technical Report AFRPL_-TR-72-88.

Levine, J. N. and Culick, F. E. C. (1974) Nonlinear analysis of solid rocket combustion instability. Uitrasystems, Inc. Irvine, Calif., Technical Report A FRPI -74-45.

Perry, E. H. and Culick, F. F. C. (1974) Measurement of wall heat transfer in the presence of large-amplitude combustion-driven oscillations, Comb. Sci. Technol. 9, 49.

Temkin, S. and Dobbins, R. A. (1966) Attenuation and dispersion of sound by particulate-relaxation processes, J. Acoust. Soc. Am. 40(2), 317-324.

\section{Appendix $A$}

\section{Nomenclature}

Some symbols defined in the text and used but briefly are not included here. A complete list of symbols appears in Part I

$\bar{a}^{2} \quad \bar{\gamma} p_{o} / \rho_{o}$ average speed of sound for the mixture

$A_{n}$ eqn (1.7)

$B_{n}$ eqn (1.7)

$C$ specific heat of particulate material

$C_{p}, C_{v}$ specific heats of gases

$\bar{C}_{p} \bar{C}_{{ }^{\prime}}$ specific heats of gas/particle mixture

$E_{\text {n. }}$ eqn (1.10)

$k$ complex wavenumber, $k=(\omega-i \alpha) / a_{\text {, }}$

$k$ wavenumber for longitudinal or axial modes

$k_{n}$ wavenumber for three-dimensional normal modes

I. length of chamber

$m$. mass flux of gases inward at the burning surface

$m_{b}{ }^{p}$ mass flux of particulate material inward at the hurning surface

$M_{b}$ Mach number of the gases at the edye of the combustion zone, $\bar{u}_{b} / a_{c}$.

$p$ pressitre

p. average pressure

$q$ perimeter of the chamber cross section

$R$ mass-averaged gas constant

$\vec{R}$ mass-averaged gas constant for the gas/particle mixture

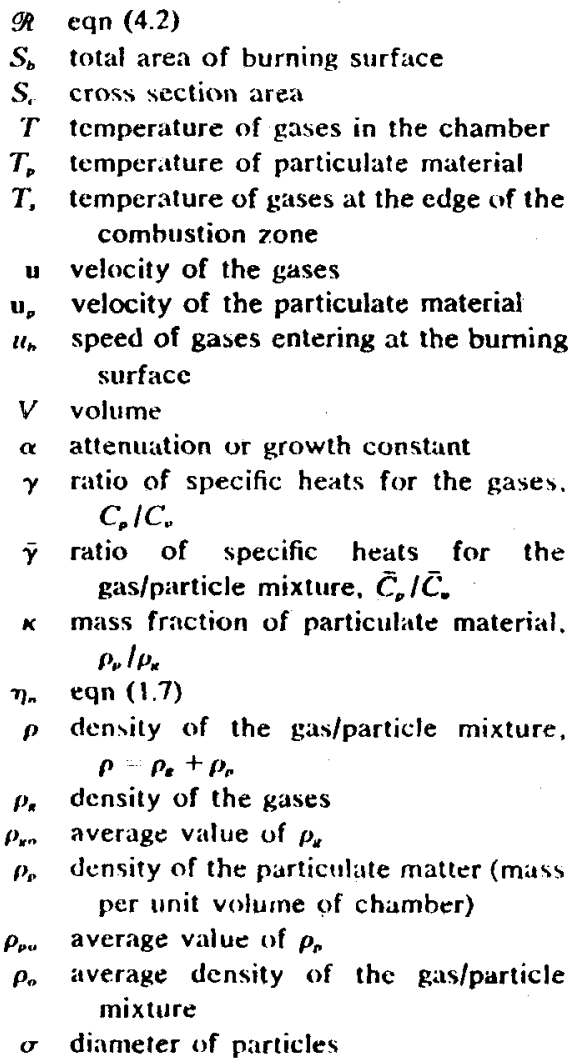


T. $\tau_{1} \cdots \mu \operatorname{sic}^{3} / 18,1$

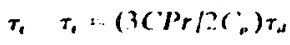

\&. momal misto shapes for ane dimensional problems

$\psi_{n}$ normal mole sliapes for three. dimensional problems
(1) anpulan frequency for one-dimensional momal anades

(1) anpular fiequency for theedimensionat normal modes

$\Omega, 1, \Omega, \quad \Omega \Omega_{d}, \Omega \Omega$, wr. 